\title{
Editorial
}

\section{Advances in Measurement Techniques for Turbomachinery Flow, Heat Transfer, and Acoustics}

\author{
Nekkanti Sitaram, ${ }^{1}$ Giovanni Maria Carlomagno, ${ }^{2}$ \\ Takayuki Matsunuma, ${ }^{3}$ and Mark McQuilling ${ }^{4}$ \\ ${ }^{1}$ IIT Madras, Chennai 600036, India \\ ${ }^{2}$ University of Naples Federico II, Piazzale Tecchio 80, 80125 Naples, Italy \\ ${ }^{3}$ National Institute of Advanced Industrial Science and Technology (AIST), 1-2-1 Namiki, Tsukuba, Ibaraki 305-8564, Japan \\ ${ }^{4}$ Parks College of Engineering, Aviation, and Technology, Saint Louis University, St. Louis, MO 63103, USA
}

Correspondence should be addressed to Nekkanti Sitaram; nsitaram.iitm@gmail.com

Received 11 March 2015; Accepted 11 March 2015

Copyright (C) 2015 Nekkanti Sitaram et al. This is an open access article distributed under the Creative Commons Attribution License, which permits unrestricted use, distribution, and reproduction in any medium, provided the original work is properly cited.

Flow in turbomachines is highly three-dimensional and complex. The flow may be incompressible or compressible subsonic/supersonic or mixed flow. The flow is further complicated by high temperatures of working fluids. Large gradients of pressure, velocity, angle, temperature, and density in all directions and with time occur in most of turbomachinery. Two-phase flow is also encountered in many turbomachines. Although CFD is being extensively used to predict flow in turbomachines, it is essential that CFD results must be validated experimentally. A wide variety of measurement techniques are required starting with simple performance measurements to most complex measurements in the rotating blade rows of turbomachines. Substantial advances are being made in different techniques used for turbomachinery flow, heat transfer, and acoustics measurements. The present special issue addresses these advances for the use of researchers in academia, industry, and R\&D laboratories.

This special issue compiles various original research articles that describe advanced measurement techniques and their application to different types of measurements in turbomachines. J. Town and C. Camci developed a time efficient adaptive gridding approach for use with a subminiature fivehole probe in an axial flow turbine. Their approach increases the possible number of measurement points in a two-hour period by $160 \%$. Flow structures behind the NGV measurement plane are identified with high spatial resolution and reduced uncertainty. R. Jangir et al. developed a subminiature four-hole probe with minimum spatial errors. Measurements made with this probe, a conventional five-hole probe and a miniature pitot probe across a calibration section, demonstrated that the errors due to gradient and surface proximity for this probe are considerably reduced compared to the fivehole probe. N. Sitaram and K. Srikanth addressed the effect of chamfer angle on the calibration curves of a large five-hole probe. They concluded that five-hole probe with a chamfer angle of $30^{\circ}$ has larger operating range, while five-hole probe with a chamfer angle of $50^{\circ}$ has increased sensitivity.

T. Astarita et al. summarized their experience in using IR thermography for convective heat transfer coefficient measurements in gas turbine cooling applications. D. Chandran and B. Prasad used IR thermography on the leading edge region of a typical gas turbine $\mathrm{NGV}$, cooled by a combination of impingement and showerhead film cooling. They found good agreement between computational and experimental results. W. L. Murray III and N. L. Key developed data processing techniques needed to detect rotor blade vibration in a forced response condition from stationary fast response pressure transducers to allow for detection of rotor vibration from transient data and lead to techniques for vibration monitoring in gas turbines. They used these techniques to detect engine order resonant response of an embedded bladed disk in a three-stage intermediate speed axial compressor. R. A. Berdanier and N. L. Key measured circumferential variation of compressor rotor tip clearance using capacitance probe 
instrumentation in the same compressor at different loading levels and at several compressor operating speeds. They found that thermal variations and centrifugal effects related to rotational speed changes affect clearance heights relative to the assembled configuration.

It is hoped that this special issue will provide useful information to wide readership from academia, industry, and research establishments and inspire development of new and more efficient measurement techniques for use in turbomachinery and other complex aerodynamics flows.

\section{Acknowledgments}

We thank the authors for their contributions. We are grateful to the reviewers who helped us during the reviewing process and selection of the papers.

Nekkanti Sitaram Giovanni Maria Carlomagno Takayuki Matsunuma Mark McQuilling 

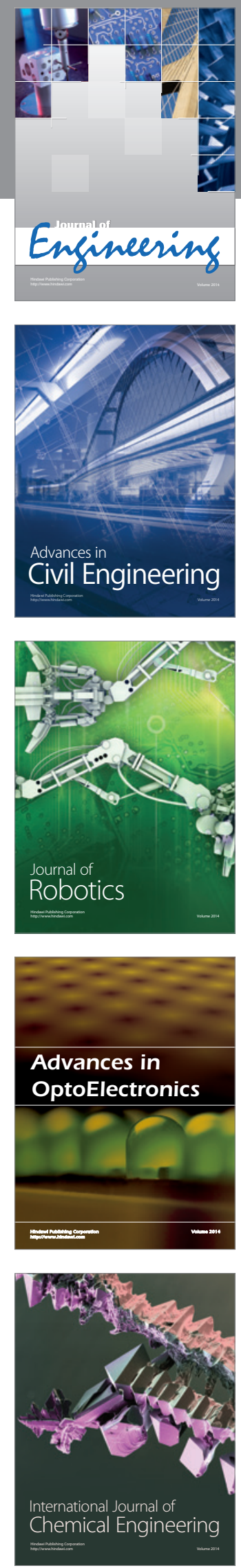

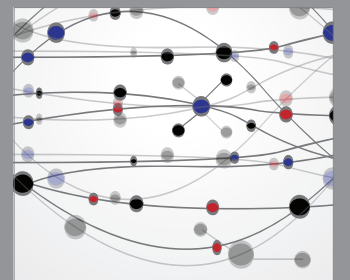

The Scientific World Journal
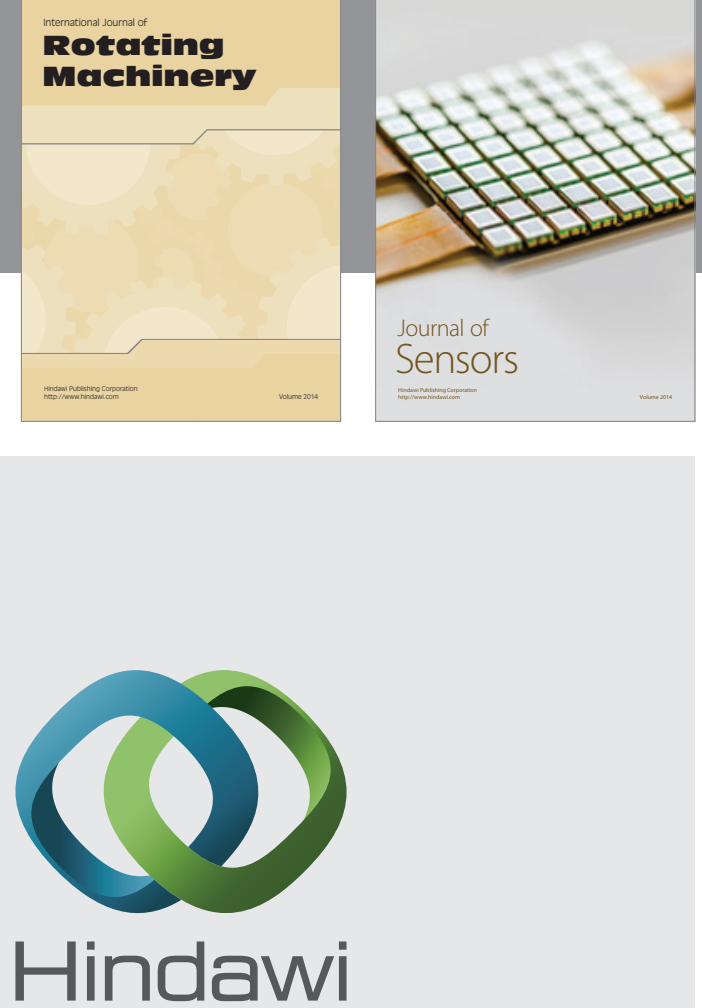

Submit your manuscripts at http://www.hindawi.com
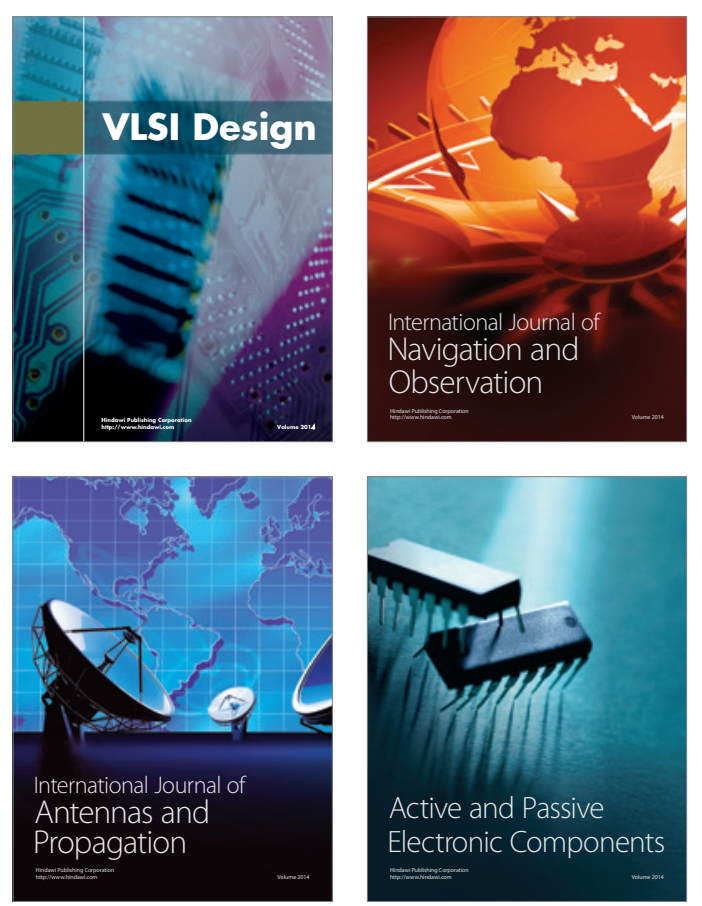
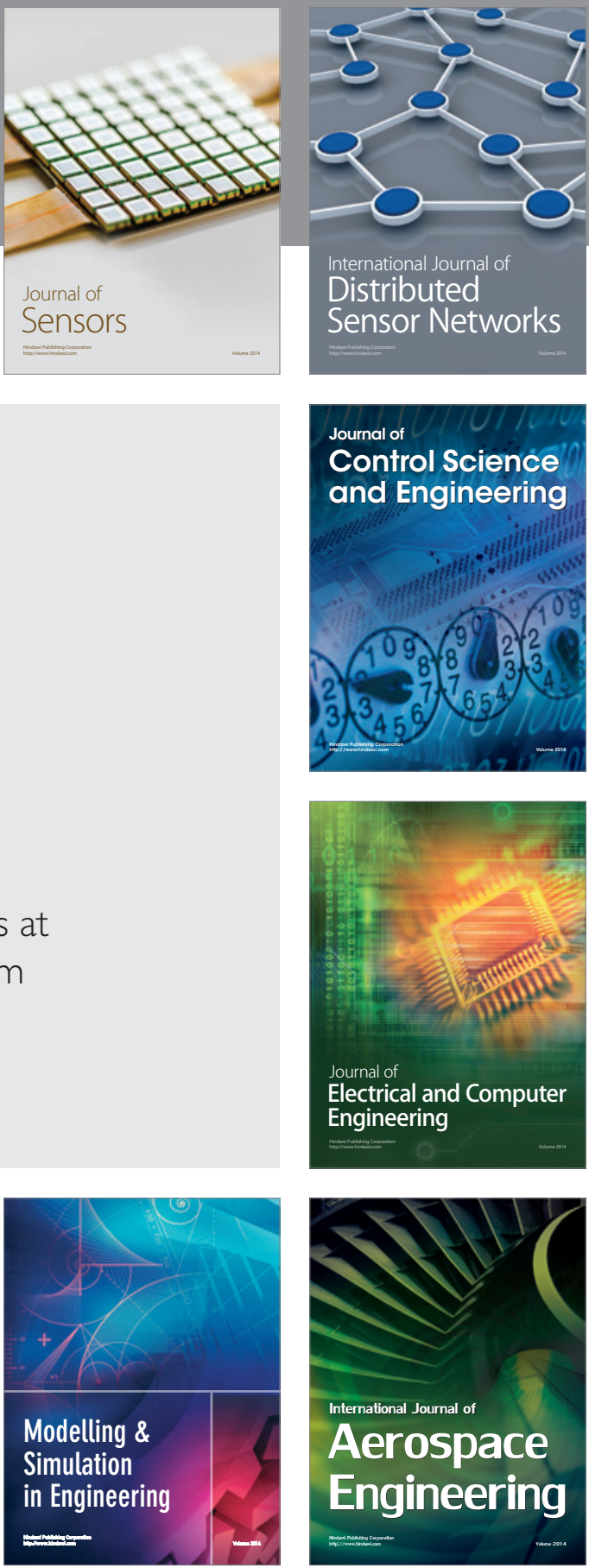

Journal of

Control Science

and Engineering
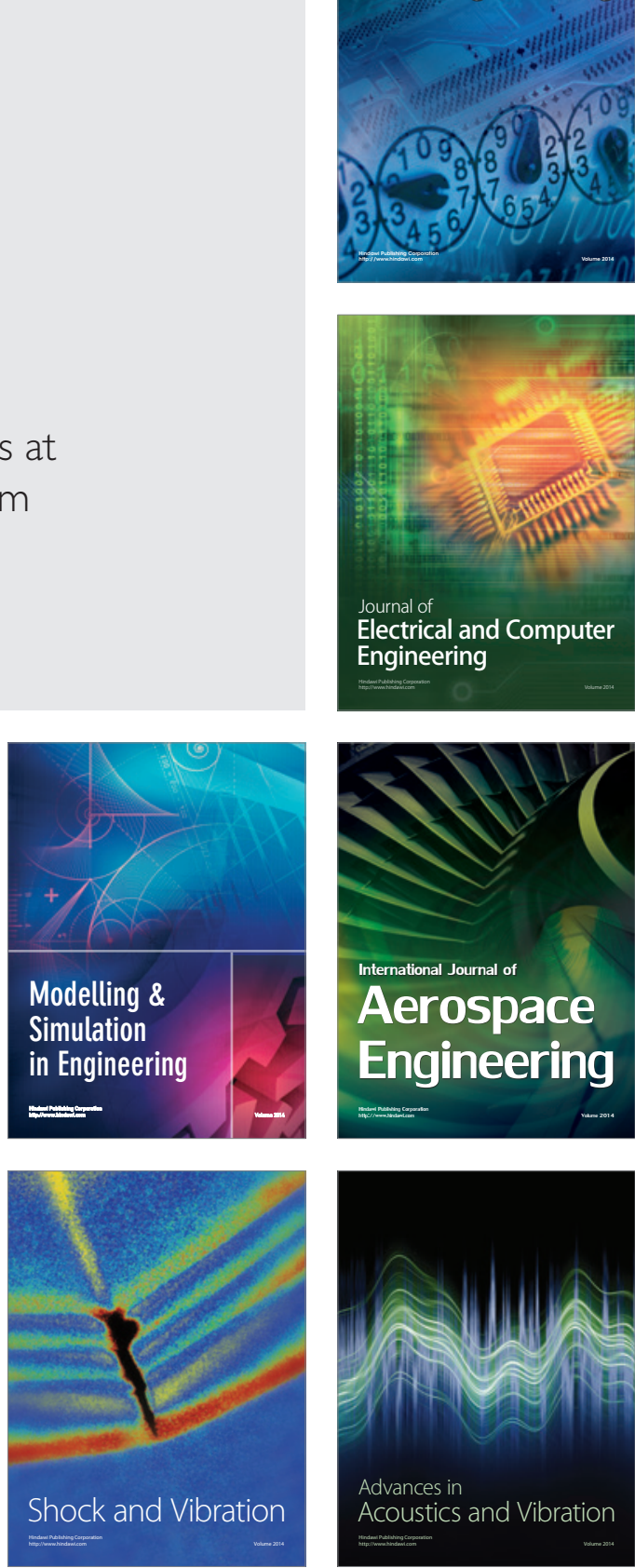